\title{
Kybernetika
}

\section{Emel Aşı1c1}

An extension of the ordering based on nullnorms

Kybernetika, Vol. 55 (2019), No. 2, 217-232

Persistent URL: http://dml.cz/dmlcz/147833

\section{Terms of use:}

(C) Institute of Information Theory and Automation AS CR, 2019

Institute of Mathematics of the Czech Academy of Sciences provides access to digitized documents strictly for personal use. Each copy of any part of this document must contain these Terms of use.

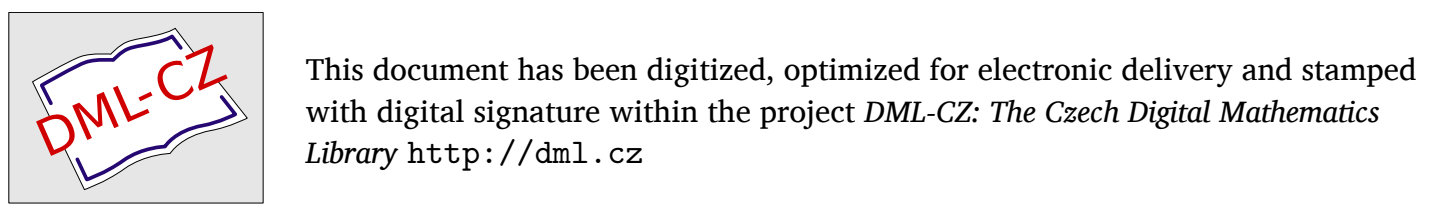




\title{
AN EXTENSION OF THE ORDERING BASED ON NULLNORMS
}

\author{
EMEL AşıII
}

In this paper, we generally study an order induced by nullnorms on bounded lattices. We investigate monotonicity property of nullnorms on bounded lattices with respect to the $F$ partial order. Also, we introduce the set of incomparable elements with respect to the F-partial order for any nullnorm on a bounded lattice. Finally, we investigate the relationship between the order induced by a nullnorm and the distributivity property for nullnorms.

Keywords: nullnorm, partial order, bounded lattice, distributivity

Classification: 03E72, 03B52

\section{INTRODUCTION}

Nullnorms and t-operators were introduced by Calvo, De Baets, Fodor [8] and Mas, Mayor, Torrens [22, respectively. Nullnorms are a generalization of triangular norms (t-norms, for short) and triangular conorms (t-conorms, for short) that are useful tool in many different fields, such as expert systems, neural networks and fuzzy logic. Nullnorms allow the freedom for the zero element $a$ (sometimes called absorbing) to be an arbitrary element from the unit interval [0,1], which is 0 for t-norms and 1 for t-conorms. And then Mas, Mayor and Torrens 23] have shown that nullnorms and t-operators are equivalent since they have the same block structures in $[0,1]^{2}$. The equivalence between nullnorms and t-operators is valid on $[0,1]$.

Karaçal, Ince and Mesiar [19] studied nullnorms on bounded lattices. They shown the existence of nullnorms with zero element $a$ for an arbitrary element $a \in L \backslash\{0,1\}$ with underlying t-norms and t-conorms on an arbitrary bounded lattice. They obtained the smallest and the greatest nullnorm on a bounded lattice.

Karaçal and Kesicioğlu [20] introduced a partial order defined by means of t-norms.

Also, Aşıcı [1] defined an order induced by a nullnorm on a bounded lattice $L$ and investigated some properties of such an order. For more details on t-norms, t-conorms nullnorms and uninorms we refer to [2, 3, 4, 10, 11, 12, 15, 16, 17, 18, 24.

In this paper, we deeply investigate some properties of an order induced by nullnorms on bounded lattices. The paper is organized as follows. We shortly recall some basic notions in Section 2. In Section 3, we investigate monotonicity property of nullnorms

DOI: $10.14736 /$ kyb-2019-2-0217 
on bounded lattices with respect to the $F$-partial order. Also, we give a sufficient condition for the set of all idempotent elements of t-norm and t-conorm to be a lattice with respect to the $F$-partial order. In Section 4 , we define the set $\mathcal{I}_{F}{ }^{(x)}$, denoting the set of all incomparable elements with arbitrary but fixed $x \in L$ according to $F$-partial order and we investigate some properties of this introduced set. Next, we define the set of incomparable elements with respect to the $F$-partial order for any nullnorm on bounded lattices. Then, we determine sets of incomparable elements with respect to the $F$-partial order of the greatest and the smallest nullnorm on a bounded lattice. In Section 5, we investigate the relationship between the order induced by a nullnorm and the distributivity property for nullnorms on the unit interval $[0,1]$. We give our concluding remarks in Section 6.

\section{PRELIMINARIES}

Let us now recall all necessary basic notions. A bounded lattice $(L, \leq)$ is a lattice which has the top and bottom elements, which are written as 1 and 0 , respectively, that is there exist two elements $1,0 \in L$ such that $0 \leq x \leq 1$, for all $x \in L$.

Definition 2.1. (Calvo et al. 8], Karaçal et al. 19]) A nullnorm is a binary operator $F: L^{2} \rightarrow L$ which is commutative, associative, non-decreasing in each variable and there exists some element $a \in L$ such that $F(x, 0)=x$ for all $x \leq a, F(x, 1)=x$ for all $x \geq a$.

Clearly, $F$ is a t-norm if $a=0$ and a t-conorm $a=1$. It is easy to show that $F(x, a)=a$ for all $x \in L$. Therefore, $a \in L$ is the zero (absorbing) element for $F$. It is clear that $F(1,0)=a$.

In this study, for the sake of brevity, the set $[0, a) \times(a, 1] \cup(a, 1] \times[0, a)$ for $a \in L \backslash\{0,1\}$ is denoted by $D_{a}$, i. e., $D_{a}=[0, a) \times(a, 1] \cup(a, 1] \times[0, a)$ for $a \in L \backslash\{0,1\}$.

Example 2.2. (Klement et al. [21]) The following are the four basic t-norms and tconorms on $[0,1]$ given by, respectively,

$T_{M}(x, y)=\min (x, y)$

$T_{P}(x, y)=x y$

$S_{M}(x, y)=\max (x, y)$

$T_{L}(x, y)=\max (x+y-1,0)$

$S_{P}(x, y)=x+y-x y$

$S_{L}(x, y)=\min (x+y, 1)$

$T_{D}(x, y)= \begin{cases}0 & (x, y) \in[0,1)^{2} \\ \min (x, y) & \text { otherwise }\end{cases}$

$S_{D}(x, y)= \begin{cases}1 & (x, y) \in(0,1]^{2} \\ \max (x, y) & \text { otherwise }\end{cases}$

Extremal t-norms $T_{\wedge}$ and $T_{W}$ on $L$ are defined as follows, respectively:

$T_{\wedge}(x, y)=x \wedge y$

$T_{W}(x, y)= \begin{cases}x & y=1, \\ y & x=1, \\ 0 & \text { otherwise. }\end{cases}$

Similarly, the t-conorms $S_{\vee}$ and $S_{W}$ can be defined as above.

In particular, $T_{W}=T_{D}$ and $T_{\wedge}=T_{M}$ for $L=[0,1]$. 
Example 2.3. (Klement et al. [21]) The t-norm $T^{n M}$ on $[0,1]$ is defined as follows:

$$
T^{n M}(x, y)= \begin{cases}0 & x+y \leq 1 \\ \min (x, y) & \text { otherwise }\end{cases}
$$

is called nilpotent minimum t-norm.

The t-norm $T^{*}$ on $[0,1]$ is defined as follows:

$$
T^{*}(x, y)=\left\{\begin{array}{lll}
0 & (x, y) \in(0, k)^{2}, \\
\min (x, y) & \text { otherwise. } & 0<k<1
\end{array}\right.
$$

Definition 2.4. (Casasnovas and Mayor [9]) A t-norm $T$ on $L$ is divisible if the following condition holds:

$$
\forall x, y \in L \quad \text { with } \quad x \leq y \quad \text { there is a } z \in L \text { such that } \quad x=T(y, z) .
$$

Definition 2.5. (Birkhoff [7]) Given a bounded lattice $(L, \leq, 0,1)$ and $a, b \in L$. If $a$ and $b$ are incomparable, in this case we use the notation $a \| b$.

Definition 2.6. (Drewniak et al. 14]) An element $x \in L$ is called an idempotent element of a function $F: L^{2} \rightarrow L$ if $F(x, x)=x$. The function $F$ is called idempotent if all elements of $L$ are idempotent.

Definition 2.7. (Karaçal and Kesicioğlu 20] ) Let $L$ be a bounded lattice and $T$ be a t-norm on $L$. The order defined as follows is called a $T$ - partial order (triangular order) for t-norm $T$ :

$$
x \preceq_{T} y: \Leftrightarrow T(\ell, y)=x \text { for some } \ell \in L .
$$

Definition 2.8. (Aşıcı [1]) Let $(L, \leq, 0,1)$ be a bounded lattice and $F$ be a nullnorm with zero element $a$ on $L$. Define the following relation, for $x, y \in L$, as

$$
x \preceq_{F} y: \Leftrightarrow\left\{\begin{array}{l}
\text { if } x, y \in[0, a] \text { and there exist } k \in[0, a] \text { such that } F(x, k)=y \text { or, } \\
\text { if } x, y \in[a, 1] \text { and there exist } \ell \in[a, 1] \text { such that } F(y, \ell)=x \text { or, } \\
\text { if }(x, y) \in L^{*} \text { and } x \leq y
\end{array}\right.
$$

where $I_{a}=\{x \in L \mid x \| a\}$ and $L^{*}=[0, a] \times[a, 1] \cup[0, a] \times I_{a} \cup[a, 1] \times I_{a} \cup[a, 1] \times[0, a] \cup$ $I_{a} \times[0, a] \cup I_{a} \times[a, 1] \cup I_{a} \times I_{a}$.

Note: The partial order $\preceq_{F}$ in $(1)$ is called $F$-partial order on $L$.

Lemma 2.9. (Aşıcı 1]) Let $(L, \leq, 0,1)$ be a bounded lattice. For all nullnorms $F$ and all $x \in L$ it holds that $0 \preceq_{F} x, x \preceq_{F} x$ and $x \preceq_{F} 1$.

Proposition 2.10. (Aşıcı [1]) Let $(L, \leq, 0,1)$ be a bounded lattice and $F$ be a nullnorm on $L$. If $x \preceq_{F} y$ for any $x, y \in L$, then $x \leq y$. 


\section{ON THE F-PARTIAL ORDER}

In this section, we investigate monotonicity property of nullnorms on bounded lattices with respect to the $F$-partial order. Also, we give a sufficient condition for the set of all idempotent elements of t-norm and t-conorm to be a lattice with respect to the $F$-partial order.

Proposition 3.1. Let $(L, \leq, 0,1)$ be a bounded lattice, $F$ be a nullnorm with zero element $a$ on $L$ and $a$ is comparable with all elements of $L$. If $x \preceq_{F} y$ for $x, y \in L$, then $F(x, z) \preceq_{F} F(y, z)$ for all $z \in L$.

Proof. Let $x \preceq_{F} y$ for $x, y \in[0, a]$. Then there exists an element $k \in[0, a]$ such that $F(x, k)=y$.

$$
F(y, z)=F(F(x, k), z)=F(k, F(x, z)) .
$$

Since $x \leq a$, then it must be the case that $F(x, z) \leq F(a, z)=a$ for all $z \in L$. It is clear that $F(y, z) \leq a$. So, $F(x, z) \preceq_{F} F(y, z)$.

Let $x \preceq_{F} y$ for $x, y \in[a, 1]$. Then there exists an element $\ell \in[a, 1]$ such that $F(y, \ell)=x$.

$$
F(x, z)=F(F(y, \ell), z)=F(\ell, F(y, z)) .
$$

Since $y \geq a$, then it must be the case that $F(y, z) \geq F(a, z)=a$ for all $z \in L$. It is clear that $F(x, z) \geq a$. So, $F(x, z) \preceq_{F} F(y, z)$.

Finally, let $x \preceq_{F} y$ for $x, y \notin[0, a]$ and $x, y \notin[a, 1]$. Due to the fact that $a$ is comparable with all elements of $L$ and $x \preceq_{F} y$, then we have that $x \leq a \leq y$. So, we get that $F(x, z) \leq F(a, z)=a \leq F(y, z)$. Hence, $F(x, z) \preceq_{F} F(y, z)$ by the definition of $\preceq F$.

Corollary 3.2. Let $(L, \leq, 0,1)$ be a bounded lattice, $F$ be a nullnorm with zero element $a$ on $L$ and $a$ is comparable with all elements of $L$. If $\left(L, \preceq_{F}\right)$ is a lattice, then $F:\left(L, \preceq_{F}\right)^{2} \rightarrow\left(L, \preceq_{F}\right)$ is a nullnorm with zero element $a$.

Corollary 3.3. (Karaçal and Kesicioğlu [20]) Let $(L, \leq, 0,1)$ be a bounded lattice, $T$ be a t-norm on $L$. If $\left(L, \preceq_{T}\right)$ is a lattice, then $T:\left(L, \preceq_{T}\right)^{2} \rightarrow\left(L, \preceq_{T}\right)$ is a t-norm on $L$.

Proposition 3.4. (Karaçal et al. [19]) Let $(L, \leq, 0,1)$ be a bounded lattice, $a \in$ $L \backslash\{0,1\}$ and $F$ be a nullnorm with zero element $a$ on $L$. Then

(i) $S^{*}=\left.F\right|_{[0, a]^{2}}:[0, a]^{2} \rightarrow[0, a]$ is a t-conorm on $[0, a]$.

(ii) $T^{*}=\left.F\right|_{[a, 1]^{2}}:[a, 1]^{2} \rightarrow[a, 1]$ is a t-norm on $[a, 1]$.

Proposition 3.5. (Karaçal and Kesicioğlu [20]) Let $(L, \leq, 0,1)$ be a bounded lattice, $F$ be a nullnorm with zero element $a$ on $L$. If $\left([0, a] \cup[a, 1], \preceq_{F}\right)$ is a chain, then $S^{*}$ and $T^{*}$ are divisible on $[0, a]$ and $[a, 1]$, respectively, i. e., $\preceq_{F}=\leq$.

Remark 3.6. The converse of the above Proposition 3.5 may not be true. Here is an example illustrating such a case. 


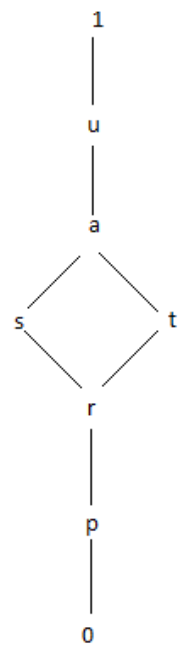

Fig. 1. The order $\leq$ on $L$.

Example 3.7. Consider the lattice $(L=\{0, p, r, s, t, a, u, 1\}, \leq, 0,1)$ whose lattice diagram is displayed in Figure 1 .

Consider the nullnorm $F: L^{2} \rightarrow L$ with zero element $a$ defined as follows [19:

$$
F(x, y)= \begin{cases}x \vee y & (x, y) \in[0, a]^{2} \\ y & x=1, y \geq a \\ x & y=1, x \geq a \\ a & \text { otherwise }\end{cases}
$$

It is clear that $S_{\vee}(x, y)=x \vee y$ is a divisible t-conorm on $[0, a]$. But $\left([0, a], \preceq_{F}\right)$ is not a chain.

Note: In this paper, for any subset $X$ of $L, \bar{X}_{\preceq_{F}}\left(\underline{X}_{\preceq_{F}}\right)$ denotes the set of the upper (lower) bounds of $X$ with respect to $\preceq_{F}$. Also, for any $x, y \in L, x \wedge_{F} y\left(x \vee_{F} y\right)$ denotes the greatest (least) element of the lower (upper) bounds with respect to $\preceq_{F}$, if they exist.

Proposition 3.8. Let $(L, \leq, 0,1)$ be a bounded lattice, $F$ be a nullnorm with zero element $a$ on $L$ such that $a$ is comparable with all elements of $L$ and $H_{S^{*}}$ and $H_{T^{*}}$ be the sets of all idempotent elements of $S^{*}$ and $T^{*}$, respectively. If $S^{*}$ is $\wedge$-distributive and $T^{*}$ is $\vee$-distributive, then $\left(H_{S^{*}} \cup H_{T^{*}}, \preceq_{F}\right)$ is a bounded lattice.

Proof. Let $x, y \in H_{S^{*}} \cup H_{T^{*}}$. We assume that $x, y \in H_{S^{*}}$. Firstly, we want to show $x \vee_{F} y=F(x, y)$. 
Since $F(x, y)=F(x, y)$, it must be $x \preceq_{F} F(x, y)$ and $y \preceq_{F} F(x, y)$. So, it holds $F(x, y) \in \overline{\{x, y\}}_{\prec_{F}}$. Let $k \in \overline{\{x, y\}}_{\prec_{F}}$ be arbitrary. Then it must be the case that $x \preceq_{F} k$ and $y \preceq_{F} k$. Since $a$ is comparable with all elements of $L$, either $k \leq a$ or $a \leq k$.

Let $k \leq a$. Then there exists elements $k_{1}, k_{2} \in[0, a]$ such that

$k=F\left(x, k_{1}\right)=\left.F\right|_{[0, a]^{2}}\left(x, k_{1}\right)=S^{*}\left(x, k_{1}\right)$ and $k=F\left(y, k_{2}\right)=\left.F\right|_{[0, a]^{2}}\left(y, k_{2}\right)=$ $S^{*}\left(y, k_{2}\right)$.

Then we have that

$$
\begin{gathered}
F(k, x)=\left.F\right|_{[0, a]^{2}}(k, x)=S^{*}\left(S^{*}\left(x, k_{1}\right), x\right)=S^{*}\left(k_{1}, S^{*}(x, x)\right)=S^{*}\left(k_{1}, x\right)=k . \\
F(k, y)=\left.F\right|_{[0, a]^{2}}(k, y)=S^{*}\left(S^{*}\left(y, k_{2}\right), y\right)=S^{*}\left(k_{2}, S^{*}(y, y)\right)=S^{*}\left(k_{2}, y\right)=k .
\end{gathered}
$$

Thus, we obtain that

$$
F(k, F(x, y))=F(F(k, x), y)=F(k, y)=k .
$$

So, it holds $F(x, y) \preceq_{F} k$.

Let $a \leq k$. Since $x, y \leq a$, it is clear that $F(x, y) \leq a$. Since $F(x, y) \leq a$ and $a \leq k$, we get that $F(x, y) \preceq_{F} k$, by the definition of $\preceq_{F}$. Thus, it is obtained $x \vee_{F} y=F(x, y)$.

Now, we will show that $x \wedge_{F} y=x \wedge y$.

It is clear that

$$
\begin{gathered}
S^{*}(x \wedge y, x \wedge y) \leq S^{*}(x, x)=x \text { and } S^{*}(x \wedge y, x \wedge y) \leq S^{*}(y, y)=y . \\
x \wedge y \leq S^{*}(x \wedge y, x \wedge y) \leq x \wedge y .
\end{gathered}
$$

We have that $S^{*}(x \wedge y, x \wedge y)=x \wedge y$. So, $x \wedge y \in H_{S^{*}}$. And

$$
x=S^{*}(x, 0) \leq S^{*}(x, x \wedge y) \leq S^{*}(x, x)=x .
$$

So, it is obtained that

$$
x=S^{*}(x, x \wedge y)=\left.F\right|_{[0, a]^{2}}(x, x \wedge y)=F(x, x \wedge y) .
$$

Hence, we get that $x \wedge y \preceq F x$.

$$
y=S^{*}(y, 0) \leq S^{*}(y, x \wedge y) \leq S^{*}(y, y)=y .
$$

And it is that

$$
y=S^{*}(y, x \wedge y)=\left.F\right|_{[0, a]^{2}}(y, x \wedge y)=F(y, x \wedge y) .
$$

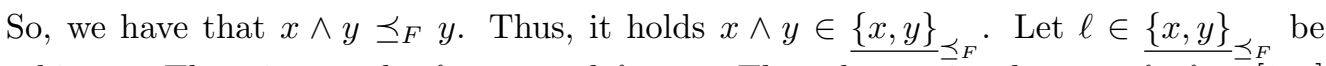
arbitrary. Then, it must be $\ell \preceq_{F} x$ and $\ell \preceq_{F} y$. Then there exist elements $\ell_{1}, \ell_{2} \in[0, a]$ such that

$$
F\left(\ell_{1}, \ell\right)=\left.F\right|_{[0, a]^{2}}\left(\ell_{1}, \ell\right)=S^{*}\left(\ell_{1}, \ell\right)=x \text { and } F\left(\ell_{2}, \ell\right)=\left.F\right|_{[0, a]^{2}}\left(\ell_{2}, \ell\right)=S^{*}\left(\ell_{2}, \ell\right)=y .
$$

Since $S^{*}$ is $\wedge$-distributive, we have that

$$
x \wedge y=S^{*}\left(\ell_{1}, \ell\right) \wedge S^{*}\left(\ell_{2}, \ell\right)=S^{*}\left(\ell_{1} \wedge \ell_{2}, \ell\right)=\left.F\right|_{[0, a]^{2}}\left(\ell_{1} \wedge \ell_{2}, \ell\right)=F\left(\ell_{1} \wedge \ell_{2}, \ell\right) .
$$


Since $\ell_{1} \wedge \ell_{2} \leq a$, we get that $\ell \preceq_{F} x \wedge y$. So, it is obtained $x \wedge_{F} y=x \wedge y$.

Let $x, y \in H_{T^{*}}$. Similarly we have that $x \wedge_{F} y=F(x, y)$ and $x \vee_{F} y=x \vee y$.

Let $x \in H_{S^{*}}$ and $y \in H_{T^{*}}$. Then it must be $x \leq a \leq y$. So, we have that $x \preceq_{F} y$, by the definition of $\preceq_{F}$. It is clear that $x \wedge_{F} y=x$ and $x \vee_{F} y=y$. Let $x \in H_{T^{*}}$ and $y \in H_{S^{*}}$. Then we have that $x \wedge_{F} y=y$ and $x \vee_{F} y=x$. Consequently, $\left(H_{S^{*}} \cup H_{T^{*}}, \preceq_{F}\right)$ is a lattice. Since $0 \in H_{S^{*}}$ and $1 \in H_{T^{*}},\left(H_{S^{*}} \cup H_{T^{*}}, \preceq_{F}\right)$ is a bounded lattice.

Remark 3.9. The converse of the above Proposition 3.8 may not be true. Here is an example illustrating such a case.

Example 3.10. Consider the lattice $(L=\{0, b, c, d, e, f, a, 1\}, \leq, 0,1)$ whose lattice diagram is displayed in Figure 2.

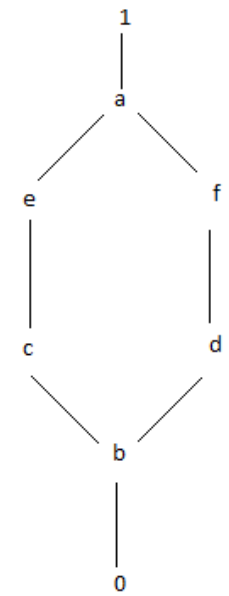

Fig. 2. The order $\leq$ on $L$.

Consider the unique idempotent nullnorm $F$ with zero element $a \in L \backslash\{0,1\}$ defined as follows [13:

$$
F(x, y)= \begin{cases}x \vee y & (x, y) \in[0, a]^{2} \\ x \wedge y & (x, y) \in[a, 1]^{2} \\ a & \text { otherwise. }\end{cases}
$$

It is clear that $S^{*}(x, y)=x \vee y$ and $T^{*}(x, y)=x \wedge y$. Since $H_{S^{*}}=\{0, b, c, d, e, f, a\}$ and $H_{T^{*}}=\{a, 1\}$, then we have $\left(H_{S^{*}} \cup H_{T^{*}}, \preceq_{F}\right)$ is a bounded lattice, but $S^{*}$ is not $\wedge$-distributive. Now, we will show this claim.

$S^{*}(e \wedge f, d)=S^{*}(b, d)=d$.

$S^{*}(e, d) \wedge S^{*}(f, d)=a \wedge f=f$.

Since $d \neq f, S^{*}$ is not $\wedge$-distributive. 
Corollary 3.11. Let $(L, \leq, 0,1)$ be a bounded lattice, $F$ be a nullnorm with zero element $a$ on $L$ such that $a$ is comparable with all elements of $L$ and $H_{S^{*}}$ and $H_{T^{*}}$ be the sets of all idempotent elements of $S^{*}$ and $T^{*}$, respectively. If $S^{*}$ is an infinitely $\wedge$-distributive and $T^{*}$ is an infinitely $\vee$-distributive, then $\left(H_{S^{*}} \cup H_{T^{*}}, \preceq_{F}\right)$ is a complete lattice.

\section{ABOUT THE SET $K_{F}^{L}$ ON ANY BOUNDED LATTICE}

Let $F$ be a nullnorm on $(L, \leq, 0,1)$ with zero element $a$ and let $I_{F}^{L(x)}$ be defined by

$$
\begin{aligned}
\mathcal{I}_{F}^{L(x)}= & \left\{y_{x} \in L \backslash\{0,1\} \mid\left[x<y_{x} \text { and } x \npreceq_{F} y_{x}\right]\right. \text { or } \\
& {\left.\left[y_{x}<x \text { and } y_{x} \npreceq_{F} x\right] \text { or } x \| y_{x}\right\} . }
\end{aligned}
$$

In the following, the notation $\mathcal{I}_{F}^{L(x)}$ is used to denote the set of all incomparable elements with $x \in L$ according to $\preceq_{F}$. Clearly, $\mathcal{I}_{F}^{L(x)}=\emptyset$, for $x=0$ and $x=1$.

Definition 4.1. Let $(L, \leq, 0,1)$ be a bounded lattice. The set $I_{x}^{L}$ for $x \in L \backslash\{0,1\}$ is defined by

$$
I_{x}^{L}=\{y \in L \backslash\{0,1\} \mid x \| y\} .
$$

For any nullnorm on $(L, \leq, 0,1)$, we get that $I_{x}^{L} \subseteq \mathcal{I}_{F}^{L(x)}$ for $x \in L$.

Proposition 4.2. Let $(L, \leq, 0,1)$ be a bounded lattice and

$$
F^{(\wedge)}(x, y)= \begin{cases}x \wedge y & (x, y) \in[a, 1]^{2} \\ a & (x, y) \in(0, a]^{2} \cup[0, a] \times I_{a} \cup I_{a} \times[0, a] \cup D_{a} \\ (x \vee a) \wedge(y \vee a) & (x, y) \in[a, 1] \times I_{a} \cup I_{a} \times[a, 1] \cup I_{a} \times I_{a} \\ x \vee y & \text { otherwise, }\end{cases}
$$

be the greatest nullnorm on $L$ with zero element $a$ (see [19]). Then

a) $\mathcal{I}_{F(\wedge)}^{L(x)}=\left\{y_{x} \in(0, a) \quad \mid x \neq y_{x}\right\} \cup I_{x}^{L}$ for all $x \in(0, a)$.

b) $\mathcal{I}_{F(\wedge)}^{L(x)}=I_{x}^{L}$ for all $x \in(a, 1)$ or $x \| a$.

Proof. a) Let $y_{x} \in \mathcal{I}_{F^{(\wedge)}}^{L(x)}$ be arbitrary for $x \in(0, a)$. Based on Lemma 2.9 it must be $x \neq y_{x}$. Thus, we need to show that $y_{x} \in(0, a)$ or $y_{x} \in I_{x}^{L}$. We consider that $y_{x} \notin(0, a)$ and $y_{x} \notin I_{x}^{L}$. Since $y_{x} \in \mathcal{I}_{F^{(\wedge)}}^{L(x)}$, then it must be the case that $\left[y_{x}<x\right.$ and $\left.y_{x} \npreceq_{F^{(\wedge)}} x\right]$ or $\left[x<y_{x}\right.$ and $\left.x \npreceq_{F^{(\wedge)}} y_{x}\right]$ or $x \| y_{x}$.

Let $y_{x}<x$ and $y_{x} \npreceq_{F^{(\wedge)}} x$.

Since $y_{x} \notin(0, a)$, we have that $y_{x}=0$ or $y_{x} \in[a, 1)$ or $y_{x} \| a$. If $y_{x}=0$, then we have $y_{x}=0 \preceq_{F^{(\wedge)}} x$, which is a contradiction. Let $y_{x} \in[a, 1)$. Since $a \leq y_{x}<x$, we have that $F^{(\wedge)}\left(x, y_{x}\right)=x \wedge y_{x}=y_{x}$ by the definition of $F^{(\wedge)}$. So, it holds $y_{x} \preceq_{F^{(\wedge)}} x$, which is a contradiction. Since $y_{x}<x<a$, it can not be $y_{x} \| a$.

Let $x<y_{x}$ and $x \npreceq_{F(\wedge)} y_{x}$.

Since $y_{x} \notin(0, a)$, we have a similar contradiction for $y_{x}=0$ and $y_{x} \in[a, 1)$. 
Let $y_{x} \| a$. Due to the fact that $x<y_{x}$ and $y_{x} \| a$, it is obtained that $x \preceq_{F^{(\wedge)}} y_{x}$, a contradiction by the definition of $\preceq_{F}$.

Finally, since $y_{x} \notin I_{x}^{L}$, it can not be $x \| y_{x}$. So, it must be the case that $y_{x} \in(0, a)$ or $y_{x} \in I_{x}^{L}$.

Hence, we have $\mathcal{I}_{F(\wedge)}^{L(x)} \subseteq\left\{y_{x} \in(0, a) \quad \mid x \neq y_{x}\right\} \cup I_{x}^{L}$ for all $x \in(0, a)$.

Conversely, let $y_{x} \in(0, a)$ or $y_{x} \in I_{x}^{L}$ such that $x \neq y_{x}$ for $x \in(0, a)$. Let us show that $y_{x} \in \mathcal{I}_{F(\wedge)}^{L(x)}$.

Let $y_{x} \in(0, a)$ and $x \neq y_{x}$ for $x \in(0, a)$. Suppose that $y_{x} \notin \mathcal{I}_{F^{(\wedge)}}^{L(x)}$, i. e., $y_{x}<x$ and $y_{x} \preceq_{F^{(\wedge)}} x$ or $x<y_{x}$ and $x \preceq_{F^{(\wedge)}} y_{x}$.

i) Let $y_{x}<x$ and $y_{x} \preceq_{F^{(\wedge)}} x$. Then there exists an element $k \in[0, a]$ such that $F^{(\wedge)}\left(y_{x}, k\right)=x$. If $k=0$, then we have $x=y_{x}$, a contradiction. Since $k \in(0, a]$, it is obtained that

$$
F^{(\wedge)}\left(y_{x}, k\right)=x=a,
$$

a contradiction by the definition of $F^{(\wedge)}$. So, it holds $y_{x} \npreceq_{F^{(\wedge)}} x$.

ii) Let $x<y_{x}$ and $x \preceq_{F^{(\wedge)}} y_{x}$. Then we have that similar contradiction as in i).

So, $\left\{y_{x} \in(0, a) \quad \mid x \neq y_{x}\right\} \subseteq \mathcal{I}_{F^{(\wedge)}}^{L(x)}$ for all $x \in(0, a)$.

Let $y_{x} \in I_{x}^{L}$ for $x \in(0, a)$. By the definition of $\mathcal{I}_{F}^{L(x)}$, we have that $I_{x}^{L} \subseteq \mathcal{I}_{F}^{L(x)}$. So, $\left\{y_{x} \in(0, a) \mid x \neq y_{x}\right\} \cup I_{x}^{L} \subseteq \mathcal{I}_{F(\wedge)}^{L(x)}$.

Consequently, we find that $\mathcal{I}_{F^{(\wedge)}}^{L(x)}=\left\{y_{x} \in(0, a) \mid x \neq y_{x}\right\} \cup I_{x}^{L}$ for all $x \in(0, a)$.

b) Let $x \in(a, 1)$. It is clear that $I_{x}^{L} \subseteq \mathcal{I}_{F}^{L(x)}$ for every nullnorm on $L$. Conversely, let $y_{x} \in \mathcal{I}_{F^{(\wedge)}}^{L(x)}$ be arbitrary. We will show that $y_{x} \in I_{x}^{L}$. Suppose that $y_{x} \notin I_{x}^{L}$. In that case $x<y_{x}$ or $y_{x}<x$. If $x<y_{x}$, then we have

$$
x=x \wedge y_{x}=F^{(\wedge)}\left(x, y_{x}\right) .
$$

So, we have that $x \preceq_{F^{(\wedge)}} y_{x}$, which is a contradiction.

Let $y_{x}<x$. If $a<y_{x}<x$, then we get that $y_{x} \preceq_{F^{(\wedge)}} x$, a contradiction. If $y_{x}<a<x$, then it is obtained that $y_{x} \preceq_{F^{(\wedge)}} x$, a contradiction by the definition of $\preceq_{F}$. So, it must be $\mathcal{I}_{F(\wedge)}^{L(x)} \subseteq I_{x}^{L}$ for $x \in(a, 1)$. Consequently, we have $\mathcal{I}_{F^{(\wedge)}}^{L(x)}=I_{x}^{L}$ for $x \in(a, 1)$.

If $x \| a$, then similarly it can be shown that $\mathcal{I}_{F^{(\wedge)}}^{L(x)}=I_{x}^{L}$.

Corollary 4.3. Let $(L, \leq, 0,1)$ be a bounded lattice. For the infimum t-norm $T_{\wedge}$ on $L$, $\mathcal{I}_{T_{\wedge}}^{L(x)}=I_{x}^{L}$ for $x \in L$.

Proposition 4.4. Let $(L, \leq, 0,1)$ be a bounded lattice and

$$
F^{(\vee)}(x, y)= \begin{cases}x \vee y & (x, y) \in[0, a]^{2} \\ a & (x, y) \in[a, 1)^{2} \cup[a, 1] \times I_{a} \cup I_{a} \times[a, 1] \cup D_{a}, \\ (x \wedge a) \vee(y \wedge a) & (x, y) \in[0, a] \times I_{a} \cup I_{a} \times[0, a] \cup I_{a} \times I_{a} \\ x \wedge y & \text { otherwise. }\end{cases}
$$

be the smallest nullnorm on $L$ with zero element $a$ (see [19]). Then 
a) $\mathcal{I}_{F^{(\vee)}}^{L(x)}=\left\{y_{x} \in(a, 1) \quad \mid x \neq y_{x}\right\} \cup I_{x}^{L}$ for all $x \in(a, 1)$.

b) $\mathcal{I}_{F^{(\vee)}}^{L(x)}=I_{x}^{L}$ for all $x \in(0, a)$ or $x \| a$.

The proof of Proposition 4.4 is similar to the proof of Proposition 4.2 .

Corollary 4.5. Let $(L, \leq, 0,1)$ be a bounded lattice and $\operatorname{card}(L)>3$. For the weakest t-norm $T_{W}$ on $L, \mathcal{I}_{T_{W}}^{L(x)}=L \backslash\{0,1\}$ for $x \in L$.

Definition 4.6. Let $F$ be a nullnorm on $(L, \leq, 0,1)$ with zero element $a$ and let $K_{F}^{L}$ be defined by

$$
\begin{gathered}
K_{F}^{L}=\left\{x \in L \backslash\{0,1\} \mid \text { for some } y \in L \backslash\{0,1\},\left[x<y \text { implies } x \npreceq_{F} y\right]\right. \text { or } \\
\left.\left[y<x \text { implies } y \npreceq_{F} x\right] \text { or } x \| y\right\} .
\end{gathered}
$$

Definition 4.7. (Aşıcı and Karaçal [6]) Let $(L, \leq, 0,1)$ be a bounded lattice. The set $I_{L}$ is defined by

$$
I_{L}=\{x \in L \mid \exists y \in L \text { such that } x \| y\} .
$$

Proposition 4.8. Let $(L, \leq, 0,1)$ be a bounded lattice, $F^{(\wedge)}$ be the greatest nullnorm 2 with zero element $a$ on $L$ and $\operatorname{card}([0, a])>3$. Then $K_{F^{(\wedge)}}^{L}=(0, a) \cup I_{L}$.

Proof. Let $x \in(0, a) \cup I_{L}$. Then it must be $x \in(0, a)$ or $x \in I_{L}$. We will show that $x \in K_{F(\wedge)}^{L}$.

Let $x \in(0, a)$ and $y \in(0, a)$ such that $x<y$. Then we get that $x \npreceq_{F(\wedge)} y$. Suppose that $x \preceq_{F^{(\wedge)}} y$. Then there exists an element $k \in[0, a]$ such that

$$
F^{(\wedge)}(x, k)=y
$$

If $k=0$, then we have that $x=y$, which is a contradiction.

If $k \in(0, a]$, then $F^{(\wedge)}(x, k)=y=a$, which is a contradiction. Since for any $x \in(0, a)$, then there exists an element $y \in(0, a), x<y$ such that $x \npreceq_{F^{(\wedge)}} y, x \in K_{F^{(\wedge)}}^{L}$. Hence, $(0, a) \subseteq K_{F(\wedge)}^{L}$.

Let $x \in I_{L}$ be arbitrary. Then there exists $y \in L$ such that $x \| y$. In that case, we get that $x \in K_{F^{(\wedge)}}^{L}$, by the definition of $K_{F}^{L}$. Thus, $I_{L} \subseteq K_{F^{(\wedge)}}^{L}$.

So, it is obtained that $(0, a) \cup I_{L} \subseteq K_{F(\wedge)}^{L}$.

Conversely, let $x \in K_{F^{(\wedge)}}^{L}$ be arbitrary. We need to show that $x \in(0, a) \cup I_{L}$. Suppose that $x \notin(0, a) \cup I_{L}$. Since $x \in K_{F^{(\wedge)}}^{L}$, then there exists an element $y \in L \backslash\{0,1\}$ such that $x<y$ and $x \npreceq_{F^{(\wedge)}} y$ or $y<x$ and $y \npreceq_{F^{(\wedge)}} x$ or $x \| y$.

Let $x<y$ and $x \npreceq_{F^{(\wedge)}} y$.

If $x=0$, then we have that $0 \preceq_{F^{(\wedge)}} y$, which is a contradiction.

Let $x \in[a, 1]$. Since $x<y$, we have that

$$
F^{(\wedge)}(x, y)=x \wedge y=x .
$$

So, it is obtained that $x \preceq_{F^{(\wedge)}} y$, which is a contradiction.

Due to the fact that $x \notin I_{L}$, it is not possible $x \| a$. 
Let $y<x$ and $y \npreceq_{F^{(\wedge)}} x$.

Let $x \in[a, 1]$. In that case, $a<y, y<a$ or $y \| a$. If $y=a$, then we have that $y=a \preceq_{F^{(\wedge)}} x$, a contradiction.

If $a<y<x$, then we have that

$$
F^{(\wedge)}(x, y)=x \wedge y=y .
$$

So, we get that $y \varsigma_{F^{(\wedge)}} x$, a contradiction.

If $y<a \leq x$, it is obtained that $y \preceq_{F^{(}(\wedge)} x$, a contradiction, by the definition of $\preceq_{F}$.

If $y \| a$, since $y<x$, we have that $y \preceq_{F(\wedge)} x$, a contradiction, by the definition of $\preceq_{F}$.

Finally, due to the fact that $x \notin I_{L}$, it can not be $x \| y$. Thus, we have that $K_{F(\wedge)}^{L} \subseteq(0, a) \cup I_{L}$.

Consequently, $K_{F(\wedge)}^{L}=(0, a) \cup I_{L}$.

Corollary 4.9. Let $(L, \leq, 0,1)$ be a bounded lattice. For the infimum t-norm $T_{\wedge}$ on $L$, $K_{T_{\wedge}}^{L}=I_{L}$.

Proposition 4.10. Let $(L, \leq, 0,1)$ be a bounded lattice, $F^{(\vee)}$ be the smallest nullnorm (3) with zero element $a$ on $L$ and $\operatorname{card}([a, 1])>3$. Then $K_{F(\vee)}^{L}=(a, 1) \cup I_{L}$.

The proof of Proposition 4.10 is similar to the proof of Proposition 4.8 .

Corollary 4.11. Let $(L, \leq, 0,1)$ be a bounded lattice. For the smallest t-norm $T_{W}$ on $L$,

$$
K_{T_{W}}^{L}= \begin{cases}\emptyset, & \text { if } \operatorname{card}(L) \leq 3 \\ L \backslash\{0,1\}, & \text { otherwise. }\end{cases}
$$

Remark 4.12. Let $(L, \leq, 0,1)$ be a chain. If $|L| \leq 4$, then it is obtained that $K_{F}^{L}=\emptyset$ for any nullnorm $F$ with zero element $a \in L \backslash\{0,1\}$.

Note that if $(L, \leq, 0,1)$ is not a chain, then the statement of Remark 4.12 may be violated. For example, let $L=\{0, x, a, 1\}$ whose lattice diagram is displayed in Figure 3 .

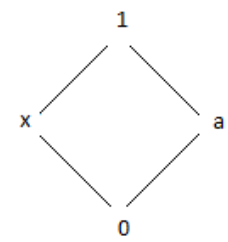

Fig. 3. The order $\leq$ on $L$.

It is clear that $K_{F}^{L} \neq \emptyset$ for every nullnorm $F$ with zero element $a$. 
Definition 4.13. Define a relation $\beta$ on the class of all nullnorms on $L$ by $F_{1} \beta F_{2}$,

$$
F_{1} \beta F_{2}: \Leftrightarrow K_{F_{1}}^{L}=K_{F_{2}}^{L}
$$

Lemma 4.14. The relation $\beta$ given in Definition 4.13 is an equivalence relation.

Proposition 4.15. Let $(L, \leq, 0,1)$ be a bounded lattice and $F_{1}$ and $F_{2}$ be two nullnorms on $L$ with zero elements $a$. If $\preceq_{F_{1}} \subseteq{ }_{F_{2}}$, then $K_{F_{2}}^{L} \subseteq K_{F_{1}}^{L}$.

Proof. Let $F_{1}$ and $F_{2}$ be two nullnorms on $(L, \leq, 0,1)$ with zero elements $a$ and $\preceq_{F_{1}} \subseteq \preceq_{F_{2}}$. We assume that $K_{F_{2}}^{L} \nsubseteq K_{F_{1}}^{L}$. Then there exists an element $x \in K_{F_{2}}^{L}$ such that $x \notin K_{F_{1}}$. Since $x \in K_{F_{2}}$, then there exists an element $y \in L \backslash\{0,1\}$ such that $x<y$ and $x \npreceq_{F_{2}} y$ or $y<x$ and $y \npreceq_{F_{2}} x$ or $x \| y$.

Let $x<y$ and $x \npreceq F_{2} y$.

In that case, it must be $x<y<a$ or $a<x<y$. The other possible conditions as follows: $x<a<y ; x\|a, y<a ; x\| a, y>a ; x\|a, y\| a ; y\|a, x<a ; y\| a, x>a$. In that conditions, we have that $x \preceq_{F} y$, a contradiction by the definition of $\preceq_{F}$. Without loss of generality, we assume that $x<y<a$. Since $x \notin K_{F_{1}}^{L}$ and $x<y$, then we have that $x \preceq_{F_{1}} y$. Since $\preceq_{F_{1}} \subseteq \preceq_{F_{2}}$, it is obtained that $x \preceq_{F_{2}} y$, a contradiction.

The proof can be shown for $y<x$ and $y \npreceq_{F_{2}} x$ in a similar manner.

Let $x \| y$. Then it must be the case that $x \in K_{F_{1}}^{L}$. This is a contradiction by the definition of $K_{F}$. So, $K_{F_{2}}^{L} \subseteq K_{F_{1}}^{L}$

Corollary 4.16. Let $(L, \leq, 0,1)$ be a bounded lattice and $F_{1}$ and $F_{2}$ be two nullnorms on $L$ with zero elements $a$. If $\preceq_{F_{1}}=\preceq_{F_{2}}$, then $K_{F_{1}}^{L}=K_{F_{2}}^{L}$

Corollary 4.17. Let $(L, \leq, 0,1)$ be a bounded lattice and $F_{1}$ and $F_{2}$ be two nullnorms on $L$ with zero elements $a$. If $\preceq_{F_{1}}=\preceq_{F_{2}}$, then $F_{1}$ and $F_{2}$ are equivalent under the relation $\beta$.

Aşıcı and Karaçal [5] have shown that for the t-norms $T_{W}$ and $T_{\wedge}$ on $L, T_{W}$ is the order-weakest t-norm and $T_{\wedge}$ is the order-strongest t-norm, i. e., $\preceq_{T_{W}} \preceq_{T} \subseteq_{T_{\wedge}}$. Also, Aşıcı [1] has shown that for the nullnorms, it need not be that case. That is, it has been shown that smallest nullnorm on the unit interval $[0,1]$ is not order-weakest nullnorm and the greatest nullnorm on the unit interval $[0,1]$ is not order-strongest nullnorm. Nevertheless, order-weakest and order-strongest nullnorms on any bounded lattice has not been determined. The following Proposition shows that order-weakest and orderstrongest nullnorms on any bounded lattice.

Proposition 4.18. Let $(L, \leq, 0,1)$ be a bounded lattice and consider the nullnorms $F_{W}=\left(S_{W}, a, T_{W}\right)$ and $F_{\vee \wedge}=\left(S_{\vee}, a, T_{\wedge}\right)$ on $L$. Then $F_{W}$ is the order-weakest nullnorm on $L$ and $F_{\vee \wedge}$ is the order-strongest nullnorm on $L$. That is,

$$
\preceq_{F_{W}} \subseteq \preceq_{F} \subseteq \preceq_{F_{\vee \wedge}} .
$$

Proof. Let $x \preceq F_{W} y$. We assume that $x, y \in[0, a]$. So, it must be the case that $x \preceq_{S_{W}} y$. Then we have that $x \preceq_{S_{\vee}} y$ for all $x, y \in[0, a]$. 
Let $x, y \in[a, 1]$. So, we obtain $x \preceq_{T_{W}} y$. Then we get $x \preceq_{T_{\wedge}} y$ for all $x, y \in[a, 1]$.

Let $x, y \notin[0, a]$ and $x, y \notin[a, 1]$. Since $x \preceq_{F_{W}} y$, then it must be $x \leq y$. So, we have that $x \preceq_{F_{\vee}} y$ by the definition of $\preceq_{F}$. So, $\preceq_{F_{W}} \subseteq \preceq_{F} \subseteq \preceq_{F_{\vee \wedge}}$.

Corollary 4.19. Let $(L, \leq, 0,1)$ be a bounded lattice and consider the nullnorms $F_{W}=\left(S_{W}, a, T_{W}\right)$ and $F_{\vee \wedge}=\left(S_{\vee}, a, T_{\wedge}\right)$ on $L$. Then $K_{F_{\vee \wedge}} \subseteq K_{F} \subseteq K_{F_{W}}$.

Corollary 4.20. Let $(L, \leq, 0,1)$ be a bounded lattice and consider the nullnorms $F_{W}=\left(S_{W}, a, T_{W}\right)$ and $F_{\vee \wedge}=\left(S_{\vee}, a, T_{\wedge}\right)$ on $L$. Then nullnorms $F_{W}$ and $F_{\vee \wedge}$ on $L$ can not be equivalent under the relation $\beta$.

Definition 4.21. Let $F$ be a nullnorm on the unit interval $[0,1]$ with zero element $a$ and $K_{F}^{\star}$ defined by

$$
\begin{gathered}
K_{F}^{\star}=\left\{x \in K_{F} \mid\right. \\
\text { for some } y, y^{\prime} \in(0,1),\left[x<y \text { but } x \npreceq_{F} y\right] \\
\text { and } \left.\left[y^{\prime}<x \text { but } y^{\prime} \npreceq_{F} x\right]\right\} .
\end{gathered}
$$

Example 4.22. Consider the nullnorm $F^{\star}:=F_{\left(S_{M}, \frac{1}{5}, T^{\star}\right)}:[0,1]^{2} \rightarrow[0,1]$ with zero element $a=\frac{1}{5}$ defined as follows (see [21]) :

$$
F_{\left(S_{M}, \frac{1}{5}, T^{\star}\right)}(x, y)= \begin{cases}\max (x, y), & (x, y) \in\left[0, \frac{1}{5}\right]^{2} \\ \frac{1}{5}, & (x, y) \in\left(\frac{1}{5}, \frac{4}{5}\right)^{2} \cup D_{\frac{1}{5}} \\ \min (x, y), & \text { otherwise. }\end{cases}
$$

Then, $K_{F}^{\star}=\left(\frac{1}{5}, \frac{4}{5}\right)$. That is, there exist some $y, y^{\prime} \in(0,1)$ such that $x<y, x \npreceq F^{\star} y$ and $y^{\prime}<x, y^{\prime} \npreceq_{F \star} x$ for $x \in\left(\frac{1}{5}, \frac{4}{5}\right)$.

The set, denoted $K_{F}^{\star}$, allows us to introduce the next equivalence relation on the class of all nullnorms on the unit interval $[0,1]$.

Definition 4.23. Define a relation $\beta^{\star}$ on the class of all nullnorms on the unit interval $[0,1]$ by $F_{1} \beta^{\star} F_{2}$,

$$
F_{1} \beta^{\star} F_{2}: \Leftrightarrow K_{F_{1}}^{\star}=K_{F_{2}}^{\star}
$$

Lemma 4.24. The relation $\beta^{\star}$ given in Definition 4.23 is an equivalence relation.

Remark 4.25. One can wonder which nullnorms are provided $K_{F}^{\star}=K_{F}$. To illustrate this claim we shall give the following example.

Example 4.26. Consider the smallest nullnorm $F^{(\vee)}:[0,1]^{2} \rightarrow[0,1]$ with zero element $a \in(0,1)$ in Proposition 4.4. It can be shown that, for all $x \in(a, 1)$, there exist elements $y, y^{\prime} \in(a, 1)$ such that $x<y, x \npreceq_{F^{(\vee)}} y$ and $y^{\prime}<x, y^{\prime} \npreceq_{F^{(\vee)}} x$. So, $K_{F^{(\vee)}}^{\star}=(a, 1)$. Since $K_{F(\vee)}=(a, 1)$ (see [1]), we get that $K_{F(\vee)}^{\star}=K_{F(\vee)}=(a, 1)$.

Consider the greatest nullnorm $F^{(\wedge)}:[0,1]^{2} \rightarrow[0,1]$ with zero element $a \in(0,1)$ in Proposition 4.2. Similarly, it can be shown that $K_{F^{(\wedge)}}^{\star}=(0, a)$. Since $K_{F^{(\wedge)}}=(0, a)$ (see [1]), we have that $K_{F^{(\wedge)}}^{\star}=K_{F^{(\wedge)}}=(0, a)$. 


\section{DISTRIBUTIVITY FOR NULLNORMS}

Definition 5.1. (Mas et al. [23]) Let $F_{1}$ and $F_{2}$ be nullnorms on $[0,1] . F_{1}$ is distributive over $F_{2}$ if they satisfy the following condition:

$$
F_{1}\left(x, F_{2}(y, z)\right)=F_{2}\left(F_{1}(x, y), F_{1}(x, z)\right)
$$

for all $x, y, z \in[0,1]$.

Proposition 5.2. Let $F_{1}$ and $F_{2}$ be nullnorms on $[0,1]$ with the same zero elements. If $F_{1}$ is distributive over $F_{2}$, then $K_{F_{2}} \subseteq K_{F_{1}}$.

Proof. Let $F_{1}$ and $F_{2}$ be nullnorms on the unit interval $[0,1]$ and $F_{1}$ is distributive over $F_{2}$. Let $x \in K_{F_{2}}$. Then there exists an element $y \in(0,1)$ such that $x<y$ and $x \npreceq_{F_{2}} y$ or $y<x$ and $y \npreceq_{F_{2}} x$. Without loss of generality, we assume that $x<y$ and $x \npreceq_{F_{2}} y$. Suppose that $x \notin K_{F_{1}}$. Since $x<y$, then it must be case that $x \preceq_{F_{1}} y$.

Let $x, y \in[0, a]$. Then there exists an element $k \in[0, a]$ such that $F_{1}(x, k)=y$.

$$
y=F_{1}(x, k)=F_{1}\left(x, F_{2}(k, 0)\right)
$$

Since, $F_{1}$ is distributive over $F_{2}$, we get that

$$
y=F_{1}\left(x, F_{2}(k, 0)\right)=F_{2}\left(F_{1}(x, k), F_{1}(x, 0)\right)=F_{2}(y, x) .
$$

So, it is obtained that $x \preceq_{F_{2}} y$, which is a contradiction.

Similarly, it can be obtained a contradiction for $x, y \in[a, 1]$. Since $x \in K_{F_{2}}$, it can not be $x, y \notin[0, a]$ and $x, y \notin[a, 1]$. Because if $x, y \notin[0, a]$ and $x, y \notin[a, 1]$, since $x<y$, we have that $x \preceq_{F} y$, by the definition of $\preceq_{F}$.

Remark 5.3. The converse of the above Proposition 5.2 may not be true. Here is an example illustrating a such case.

Example 5.4. Consider the nullnorm $F:[0,1]^{2} \rightarrow[0,1]$ with zero element $a=\frac{1}{3}$ defined as follows (see [17] ):

$$
F(x, y)= \begin{cases}\max (x, y), & (x, y) \in\left[0, \frac{1}{3}\right]^{2} \\ \frac{3 x y-x-y+1}{2}, & (x, y) \in\left[\frac{1}{3}, 1\right]^{2} \\ \frac{1}{3}, & \text { otherwise }\end{cases}
$$

and $F^{(\wedge)}:[0,1]^{2} \rightarrow[0,1]$ with zero element $a=\frac{1}{3}$ in Proposition 4.2. It is clear that $K_{F}=\emptyset$. Also, we know that $K_{F(\wedge)}=(0, a)$ (see [1]). Although $K_{F}=\emptyset \subset(0, a)=$ $K_{F^{(\wedge)}}, F^{(\wedge)}$ is not distributive over $F$. Now, we will show this claim.

$$
F^{(\wedge)}\left(\frac{1}{2}, F\left(\frac{5}{6}, \frac{6}{7}\right)\right)=F^{(\wedge)}\left(\frac{1}{2}, \frac{61}{84}\right)=\frac{1}{2} \text { and } F\left(F^{(\wedge)}\left(\frac{1}{2}, \frac{5}{6}\right), F^{(\wedge)}\left(\frac{1}{2}, \frac{6}{7}\right)\right)=F\left(\frac{1}{2}, \frac{1}{2}\right)=\frac{3}{8} .
$$

Since $\frac{1}{2} \neq \frac{3}{8}, F^{(\wedge)}$ is not distributive over $F$.

Proposition 5.5. Let $F_{1}$ and $F_{2}$ be nullnorms on $[0,1]$ with the same zero elements. If $F_{1}$ is distributive over $F_{2}$ and $F_{2}$ is distributive over $F_{1}$, then $K_{F_{1}}=K_{F_{2}}$. 


\section{CONCLUDING REMARKS}

We have discussed and investigated some properties of an order induced by a nullnorm on bounded lattices, called $F$-partial order, generally. We have given a sufficient condition for the set of all idempotent elements of t-norm and t-conorm to be a lattice with respect to the $F$-partial order. We have defined the set $\mathcal{I}_{F}{ }^{(x)}$, denoting the set of all incomparable elements with arbitrary but fixed $x \in L \backslash\{0,1\}$ according to $F$-partial order and we have investigated some properties of this introduced set. Also, we have defined the set of incomparable elements with respect to the $F$-partial order for any nullnorm on bounded lattice and we determine this introduced set of smallest and greatest nullnorm on a bounded lattice. Following this, we have investigated the relationship between the order induced by a nullnorm and the distributivity property for nullnorms on the unit interval $[0,1]$.

\section{ACKNOWLEDGEMENT}

We are grateful to the anonymous reviewers and editors for their valuable comments which helped to improve the original version of our manuscript greatly.

(Received June 5, 2017)

\section{REFERENCES}

[1] E. Aşıcı: An order induced by nullnorms and its properties. Fuzzy Sets Syst. 325 (2017), 35-46. DOI:10.1016/j.fss.2016.12.004

[2] E. Aşıcı: On the properties of the $F$-partial order and the equivalence of nullnorms. Fuzzy Sets Syst. 346 (2018), 72-84. DOI:10.1016/j.fss.2017.11.008

[3] E. Aşıcı: Some notes on the F-partial order. In: Advances in Fuzzy Logic and Technology 2017 (J. Kacprzyk , E. Szmidt, S. Zadroźny, K. Atanassov, and M.Krawczak, eds.), IWIFSGN 2017, EUSFLAT 2017. Advances in Intelligent Systems and Computing 641, Springer, Cham, pp. 78-84. DOI:10.1007/978-3-319-66830-7_7

[4] E. Aşıcı: Some remarks on an order induced by uninorms. In: Advances in Fuzzy Logic and Technology 2017 (J. Kacprzyk, E. Szmidt, S. Zadroźny, K. Atanassov, and M.Krawczak, eds.), IWIFSGN 2017, EUSFLAT 2017. Advances in Intelligent Systems and Computing 641, Springer, Cham, pp. 69-77. DOI:10.1007/978-3-319-66830-7_7

[5] E. Aşıcı and F. Karaçal: On the T-partial order and properties. Inform. Sci. 267 (2014), 323-333. DOI:10.1016/j.ins.2014.01.032

[6] E. Aşıcı and F. Karaçal: Incomparability with respect to the triangular order. Kybernetika 52 (2016), 15-27. DOI:10.14736/kyb-2016-1-0015

[7] G. Birkhoff: Lattice Theory. Third edition. Providence, 1967. DOI:10.1090/coll/025

[8] T. Calvo, B. De Baets, and J. Fodor: The functional equations of Frank and Alsina for uninorms and nullnorms. Fuzzy Sets Syst. 120 (2001), 385-394. DOI:10.1016/s01650114(99)00125-6

[9] J. Casasnovas and G. Mayor: Discrete t-norms and operations on extended multisets. Fuzzy Sets Syst. 159 (2008), 1165-1177. DOI:10.1016/j.fss.2007.12.005 
[10] G.D. Çaylı: A characterization of uninorms on bounded lattices by means of triangular norms and triangular conorms. Int. J. General Syst. 47 (2018), 772-793. DOI:10.1080/03081079.2018.1513929

[11] G. D. Çaylı: On a new class of t-norms and t-conorms on bounded lattices. Fuzzy Sets Syst. 332 (2018), 129-143. DOI:10.1016/j.fss.2017.07.015

[12] G. D. Çaylı and P. Drygaś: Some properties of idempotent uninorms on a special class of bounded lattices. Inf. Sci. 422 (2018), 352-363. DOI:10.1016/j.ins.2017.09.018

[13] G. D. Çaylı, F. Karaçal, and R. Mesiar: On a new class of uninorms on bounded lattices. Inf. Sci. 367-368 (2016), 221-231. DOI:10.1016/j.ins.2016.05.036

[14] J. Drewniak, P. Drygaś, and E. Rak: Distributivity between uninorms and nullnorms. Fuzzy Sets Syst. 159 (2008), 1646-1657. DOI:10.1016/j.fss.2007.09.015

[15] P. Drygaś: Distributive between semi-t-operators and semi-nullnorms. Fuzzy Sets Syst. 264 (2015), 100-109. DOI:10.1016/j.fss.2014.09.003

[16] P. Drygaś: A characterization of idempotent nullnorms. Fuzzy Sets Syst. 145 (2004), 455-461. DOI:10.1016/s0165-0114(03)00259-8

[17] M. Grabisch, J.-L. Marichal, R. Mesiar, and E. Pap: Aggregation Functions. Cambridge University Press, 2009. DOI:10.1017/cbo9781139644150

[18] B. Jayaram and C. J. M. Rao: On the distributivity of implication operators over $T$ and $S$ norms. IEEE Trans. Fuzzy Syst. 12 (2004), 194-198. DOI:10.1109/tfuzz.2004.825075

[19] F. Karaçal, M. A. Ince, and R. Mesiar: Nullnorms on bounded lattices. Inf.Sci. 325 (2015), 227-236. DOI:10.1016/j.ins.2015.06.052

[20] F. Karaçal and M. N. Kesicioğlu: A T-partial order obtained from t-norms. Kybernetika 47 (2011), 300-314.

[21] E. P. Klement, R. Mesiar, and E. Pap: Triangular Norms. Kluwer Academic Publishers, Dordrecht 2000. DOI:10.1007/978-94-015-9540-7

[22] M. Mas, G. Mayor, and J. Torrens: t-operators. Int. J. Uncertain. Fuzz. Knowl.-Based Syst. 7 (1999), 31-50. DOI:10.1142/s0218488599000039

[23] M. Mas, G. Mayor, and J. Torrens: The distributivity condition for uninorms and toperators. Fuzzy Sets Syst. 128 (2002), 209-225. DOI:10.1016/s0165-0114(01)00123-3

[24] B. Schweizer and A. Sklar: Statistical metric spaces. Pacific J. Math. 10 (1960), 313-334. DOI:10.2140/pjm.1960.10.313

Emel Aşıcı, Department of Software Engineering, Faculty of Technology, Karadeniz Technical University, 61830 Trabzon. Turkey.

e-mail: emelkalin@hotmail.com 